\title{
Integrated Transcriptome Sequencing Analysis Reveals Role of miR-138-5p/ TBL1X in Placenta from Gestational Diabetes Mellitus
}

\author{
Rong Dinga,b Fei Guo a,b Yong Zhang ${ }^{a, b} \quad$ Xi-Mei Liu ${ }^{a, b} \quad$ Yu-Qian Xianga,b \\ Chen Zhang ${ }^{a, b}$ Zhi-Wei Liu ${ }^{a, b}$ Jian-Zhong Sheng ${ }^{c, d}$ He-Feng Huang ${ }^{a, b, c}$ \\ Jun-Yu Zhang ${ }^{a, b}$ Jian-Xia Fana,b
}

alnternational Peace Maternity and Child Health Hospital, School of Medicine, Shanghai Jiao Tong University, Shanghai, 'Insititute of Embryo-Fetal Original Adult Disease, School of Medicine, Shanghai Jiao Tong University, Shanghai, 'Key Laboratory of Reproductive Genetics, Ministry of Education, Zhejiang University, Hangzhou, dDepartment of Pathology and Pathophysiology, School of Medicine, Zhejiang University, Hangzhou, China

\section{Key Words}

Gestational diabetes mellitus $•$ Placenta $\cdot$ RNA sequencing $\bullet$ MicroRNA $・$ miR-138-5p $•$ TBL1X

\begin{abstract}
Background/Aims: The placenta has been suggested to play a crucial role in the pathology of gestational diabetes mellitus (GDM). Placenta-specific microRNAs (miRNAs) and the corresponding targeting genes involved in the pathology of GDM still remain to be elucidated. We aimed to identify the dysregulated miRNAs and the corresponding mRNA targets through an integrated miRNA and mRNA transcriptomic profiles analysis and investigate the role of differentially expressed miR-138-5p/TBL1X in GDM. Methods: RNA sequencing (RNA-seq) was performed in 16 placentas from GDM and control group. Differentially expressed mRNAs and miRNAs in GDM were validated by quantitative PCR (qPCR). The wound healing assay and transwell migration assay were used to analyze cell migration ability. The cell proliferation was determined by CCK8 assay. Luciferase assay was used to confirm the direct binding of the targeted TBL1X with miR-138-5p. Results: Totally, 281 mRNAs and 32 miRNAs were found to be differentially expressed in the GDM placentas. The biological relationships of the miRNA/mRNA pairs were related to cellular development and function and organ morphology. Among the aberrantly expressed molecules, we selected miR-138-5p from the bioinformatics analysis and found that miR-138-5p significantly inhibited the migration and proliferation of trophoblasts (HTR-8/SVneo) by targeting the $3^{\prime}-$ UTR of TBL $1 X$. Furthermore, the aberrant expression of miR-138-5p and TBL1X was significantly correlated with the weight
\end{abstract}

R. Ding, F. Guo and Y. Zhang contributed equally to this work.

Jian-Xia Fan, M.D

and Jun-Yu Zhang, PhD
International Peace Maternity and Child Health Hospital, School of Medicine,

Shanghai Jiao Tong University, Shanghai 200030 (China)

Tel. +86-21-64070434, E-Mail fanjianxia122@126.com; junyuzhang@hotmail.com 


\section{Cellular Physiology Cell Physiol Biochem 2018;51:630-646 \begin{tabular}{ll|l} 
DOl: 10.1159/000495319 & (0) 2018 The Author(s). Published by S. Karger AG, Basel \\
and Biochemistry & Published online: 21 November 2018 www.karger.com/cpb
\end{tabular} \\ Ding et al.: Transcriptome Profile of GDM Placenta}

of the placenta. Conclusion: We present the first integrative analysis of miRNA and mRNA expression profiles in GDM placenta and uncover a more detailed role for miR-138-5p, as well as its target TBL $1 X$ in the pathology of GDM.

(C) 2018 The Author(s)

Published by S. Karger AG, Basel

\section{Introduction}

Gestational diabetes mellitus (GDM) is defined as hyperglycemia with onset or first recognition during pregnancy [1]. Due to ethnic differences and various diagnostic criteria, the prevalence of GDM varies from $5 \%$ to $20 \%$ [2]. GDM is one of the most common metabolic complications of pregnancy and shows significant influence on both mother and offspring, including increased birth weight, caesarean section, neonatal hypoglycemia and later diabetes [3]. GDM also increases the risk of long-term complications in the offspring, such as metabolic and cardiovascular diseases [4,5]. Intrauterine hyperglycemia, as a major characteristic of GDM, affects imprinted gene expression by regulating epigenetic modification in germ cells, which increases the risk of diabetes in offspring [6].

Recent studies have indicated that the normal placental function is involved in the pathology of GDM since GDM-associated hyperglycemia can be resolved after placental delivery [7, 8],[9]. The placenta secretes a series of steroids, hormones and cytokines that are required for normal pregnancy $[10,11]$. The placental size and weight in GDM pregnancies are significantly higher than those in normal pregnancies and the elevation is thought to lead to adverse neonatal outcomes, including macrosomia [12]. It was also reported that the increased placental size gives rise to the increased concentrations of human placental lactogen (hPL), estradiol and progesterone in the maternal circulation [13, 14]. These hormones secreted by the placenta show the insulin resistance, thus dramatically inducing the occurrence of GDM [15]. Aires et al. suggested that the diabetic placenta was usually accompanied by abnormal trophoblast proliferation, apoptosis and cell cycle control [16]. However, the underlying molecular mechanism contributing to heavy placental weight remains unclear.

The functional placenta is controlled by both protein-coding genes and non-coding regions, including microRNAs (miRNAs) [17]. miRNAs are small non-coding single stranded RNAs that regulate the expression of approximately $30 \%$ of all genes in animals through translational repression or mRNA degradation [18]. Previous studies on trophoblasts have suggested that miRNAs are involved in proliferative, migratory and invasive processes [19]. Many differentially expressed miRNAs have been identified based on hybridization, qPCR, and sequencing analysis in the GDM placenta or serum [20,21]. Recently, Bari et al. performed specific mRNA sequencing with trophoblast isolated from the GDM placenta and indicated that placental trophoblasts of GDM were biologically different from those in the control group at the gene expression level [22]. Li et al. compared the miRNA expression profiles of the GDM placenta with those of the control group by microarray analysis and identified 9 deregulated miRNAs [20]. However, the aforementioned studies were focused on a single analysis of the mRNA or miRNA expression profiling. The integrated analysis of mRNA and miRNA expression in the GDM placenta, to our knowledge, has not been reported.

In this study, we present an integrative analysis of the gene expression profiles of both mRNAs and miRNAs in 16 placental tissues, including those from 8 patients with GDM and 8 controls. Our data indicated that the GDM placenta was significantly different from that of the control at both the miRNA and mRNA levels. The differentially expressed genes were significantly associated with cellular growth, proliferation and migration, suggesting the association of placental overgrowth with GDM. Moreover, we have clarified that the roles of miR-138-5p and its target gene transducin $\beta$-like protein 1 (TBL1X) in trophoblasts. 


\section{Cellular Physiology Cell Physiol Biochem 2018;51:630-646

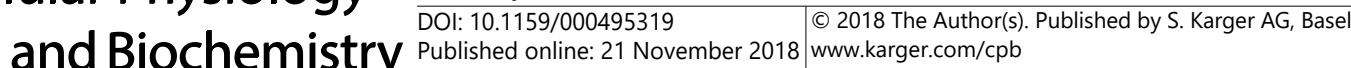 \\ Ding et al.: Transcriptome Profile of GDM Placenta}

\section{Materials and Methods}

\section{Samples}

All placenta samples were collected from the Department of Obstetrics and Gynecology of the International Peace Maternity \& Child Health Hospital (IPMCH), in the School of Medicine in Shanghai Jiao Tong University. Twenty-six normal pregnant women (control group) and 28 GDM pregnant patients (GDM group) after C-section in the third trimester of gestation were included. GDM was diagnosed by the criteria suggested by the International Association of the Diabetes and Pregnancy Study Groups (IADPSG): fasting plasma glucose $(\mathrm{PG}) \geq 5.1 \mathrm{mmol} / \mathrm{L}$ and/or 1 -hour $\mathrm{PG} \geq 10.0 \mathrm{mmol} / \mathrm{L}$ and/or 2 -hour $\mathrm{PG} \geq 8.5 \mathrm{mmol} / \mathrm{L}$. Women with in vitro fertilization (IVF), maternal diabetes history, twins (multiple) pregnancy, preeclampsia, or other pregnancy complications were excluded. All samples were dissected into fragments with the size of $\sim 1 \mathrm{~cm}^{3}$ and washed with phosphate buffered saline (PBS) immediately after elective caesarean delivery. The tissues were stored in RNAlater (Thermo Fisher Scientific, Waltham, MA, USA) at $-80{ }^{\circ} \mathrm{C}$. This study was approved by the Ethics Committee of the IPMCH. Written informed consents were obtained from all the participants.

\section{RNA sequencing and data analysis}

Total RNA was extracted from the placental tissues with the Ambion mirVana ${ }^{\text {TM }}$ PARIS ${ }^{\text {TM }}$ Kit (Thermo Fisher Scientific, Waltham, MA, USA) according to the manufacturer's instructions. The RNA sequencing (RNA-seq) library preparation has been described in detail [23]. Briefly, the Illumina TruSeq RNA Library Prep Kit (Illumina, San Diego, CA, USA) and TruSeq Small RNA Library Prep Kit (Illumina, San Diego, CA, USA) were used for the preparation of mRNA-seq and miRNA-seq libraries, respectively. All the libraries were analyzed to verify the quality and concentration with an Agilent Bioanalyzer (Agilent, Palo Alto, CA, USA). mRNA sequencing was performed on the Illumina Hiseq 2500 sequencing system (Illumina, San Diego, CA, USA) and 150-bp paired-end FASTQ read files were generated. The raw mRNA-seq data were trimmed using Trimmomatic v.0.33 [24]. Clean reads were aligned with the UCSC Homo sapiens reference genome (build hg19) using TopHat v.2.1.0 [25] followed by transcript assembly and differential transcript expression analysis with Cufflinks v.2.2.1. miRNA sequencing was performed on the Illumina HiSeq 2500 sequencing system and 50-bp single-end FASTQ reads files were generated. The raw miRNA sequencing data were trimmed with Cutadapt [26]. Clean reads were aligned with the UCSC Homo sapiens reference genome (build hg19) using the Burrows-Wheeler Aligner (BWA; v.0.7.12) [27]. The known conserved miRNAs were identified by searching miRBase (Release 21) in Homo sapiens [28]. The mapped read counts were compared in the R package DESeq for differential expression analysis [29]. mRNAs/miRNAs with absolute value fold change $\geq 2$ and $P<0.05$ were marked as significant.

\section{Functional analysis and paired miRNA-mRNA analysis}

The Ingenuity Pathway Analysis (IPA) (Qiagen, Valencia, CA, USA) was used to identify the top biological functions and canonical pathways associated with the differentially expressed mRNAs and miRNAs datasets, as described previously [30]. The Fisher's exact test was used to determine the probability that each biological function or canonical pathway assigned to the dataset is explained by chance alone. The molecular interaction networks were algorithmically generated based on the molecules' connectivity. The network scores are calculated using Fisher's exact test and are equal to the $\log _{10}(P$-value). The downstream effects analysis was performed to predict downstream biological processes and infer their activation states based on the observed gene expression changes in the dataset. A z-score was calculated and used to infer the activation states (increased or decreased) of implicated biological processes [31]. The prioritization of predicted and/or experimentally validated miRNA-mRNA target pairs was performed with the IPA miRNA target filter tool based on the expression direction and information from the Ingenuity Knowledge Base.

\section{Validation of mRNA and miRNA by $q P C R$}

Total RNA isolated from placenta was reversely transcribed with the PrimeScript ${ }^{\mathrm{TM}}$ II 1 st Strand cDNA Synthesis Kit (Takara Bio, Dalian, China) and random primers. The quantification of mRNAs was performed with the SYBR Green Kit (Takara Bio, Dalian, China) in the Applied Biosystems QuanStudio ${ }^{\mathrm{TM}} 7$ Flex (Thermo Fisher Scientific, Waltham, MA, USA). The reference gene ACTB was used as the internal control for the relative qualification of mRNA. Quantification of miRNAs was performed with miDETECT A Track ${ }^{\mathrm{TM}}$ miRNA 


\section{Cellular Physiology Cell Physiol Biochem 2018;51:630-646

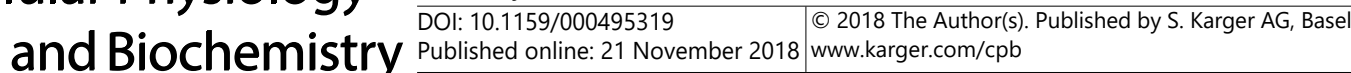 \\ Ding et al.: Transcriptome Profile of GDM Placenta}

qPCR Starter Kit (Ribobio, Guangzhou, China). U6 was used to normalize the miRNA qPCR data. The qPCR data were analyzed using the $2^{-\Delta \triangle \mathrm{Ct}}$ method normalized to ACTB or U6. The qPCR primers are listed in Table S1 (For all supplemental material see www.karger.com/10.1159/000495319/).

\section{Cell culture and transfection}

The HTR-8/SVneo cells (HTR-8, human invasive EVTs) were a generous gift from Dr. P.K. Lala (University of Western Ontario, London, Ontario, Canada). The cells were cultured in Dulbecco's modified Eagle's medium (DMEM)/F12 supplemented with 10\% fetal bovine serum (FBS) (Gibco, Grand Island, NY, USA) containing $1 \%$ antibiotics. The miR-138-5p mimic and the corresponding negative control were synthesized (Ribobio, Guangzhou, China) to investigate the effect of miR-138-5p overexpression. The miR-138-5p inhibitor and corresponding negative control were synthesized (Ribobio, Guangzhou, China) to investigate the effect of miR-138-5p inhibition. miR-138-5p mimic and miR-138-5p inhibitor were transfected into HTR-8 cells with the HiPerFect Transfection Reagent (Qiagen, Valencia, CA, USA) at a final concentration of $100 \mathrm{nM}$. To investigate the roles of TBL1X in promoting migration and proliferation of trophoblasts targeted by miR-138-5p, we cotransfected HTR-8 cells with $100 \mathrm{nM}$ miR-138-5p inhibitor and $50 \mathrm{nM}$ TBL1X siRNA (Ribobio, Guangzhou, China) to perform a rescue experiment.

\section{Wound healing assay}

The HTR-8 cells were transfected with miR-138-5p mimic, miR-138-5p inhibitor and TBL1X siRNA. When the cells reached $90 \%$ confluence after $24 \mathrm{~h}$, scratches were made with a pipette tip. To reduce the impact of cell proliferation, the cells were cultured in DMEM/F12 without FBS. After washing the cells three times with PBS, the width of the scratches was imaged and measured.

\section{Transwell migration assay}

After transfecting the HTR-8 cells with miR-138-5p mimic, miR-138-5p inhibitor and TBL1X siRNA for $24 \mathrm{~h}$, we transferred $10^{5}$ cells in $150 \mu \mathrm{L}$ of non-serum DMEM/F12 to the upper compartment of the Transwell compartments (Costar, Cambridge, MA, USA). Then, $0.6 \mathrm{~mL}$ of DMEM/F12 with 10\%FBS were added to the lower compartment. We incubated the cells in the transwell plates at $37^{\circ} \mathrm{C}$ under $\mathrm{CO}_{2}$ for $24 \mathrm{~h}$. The crystal violet-stained cells were counted under an inverted microscope.

\section{Cell proliferation assay}

The HTR-8 cells were transfected with miR-138-5p mimics or inhibitors for $24 \mathrm{~h}$. Then, cell proliferation was detected with cell counting kit-8 (CCK8, Dojindo, Japan) according to the manufacturer's instructions. Briefly, 4, 000 cells per well were seeded into 96-well plates with complete culture medium. After transfection for 12, 24, 48 and $72 \mathrm{~h}, 10 \mu \mathrm{L}$ of the CCK8 reagent were added into the testing well and incubated for $2 \mathrm{~h}$. The absorbance was measured at a wavelength of $450 \mathrm{~nm}$.

\section{Plasmid construction and luciferase assays}

For the wild-type construct, the whole length of human TBL1X 3'UTR was amplified with the following primers: forward, 5'- AAGCGATCGCGGGTTGCAGCTCTATTCTC -3' and reverse, 5'-AATGCGGCCGCTGGCTTCTTCGGTTCTTTT-3'. For the mutated construct, the mutant 3'UTR sequences without the miR-138-5p binding site were amplified with the following mutation primers: forward, 5'-GAGGAAGTGTGGTCGTCGAGGCGTGTGGATTGG-3' and reverse, 5'-CGCCTCGACGACCACCTTCCTCCTTCCCAGGC-3'. The PCR products were cloned into the downstream of the Renilla luciferase ORF in the psiCHECK-2 vector (Promega, Madison, WI, USA) at the Sgfl and NotI restriction sites.

A dual luciferase reporter assay was carried out to detect the direct interactions between miR-1385p and TBL1X. Approximately, 8000 HTR-8 cells per well were seeded into 96-well plates. In each well, we cotransfected $200 \mathrm{ng}$ of wild-type or mutant 3'UTR reporter, with miR-138-5p mimic or the corresponding negative control at a final concentration of $50 \mathrm{nM}$. The cell lysates were harvested in $24 \mathrm{~h}$ after the transfection and the luciferase activity was measured using a luciferase assay kit (Promega, Madison, WI, USA). The Renilla luciferase activity was measured with firefly luciferase as the internal control.

\section{KARGER}




\section{Cellular Physiology Cell Physiol Biochem 2018;51:630-646

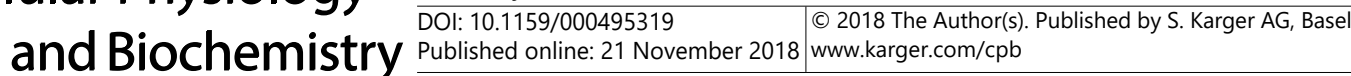 \\ Ding et al.: Transcriptome Profile of GDM Placenta}

Western blot analysis

Total protein from the placental tissues and HTR-8 cells was prepared in lysis buffer with proteinase inhibitors (Roche, Penzberg, Germany). A Pierce BCA protein assay kit (Pierce Biotechnology, Waltham, MA, USA) was used to calculate the protein concentrations. Equal amounts of protein samples were resolved in 10\% SDS-PAGE and transferred to polyvinylidene difluoride (PVDF) membranes (Bio-Rad Laboratories, Richmond, CA, USA). The membranes were separately incubated with the primary antibodies against TBL1X (Sigma, USA, 1:500) and $\beta$-actin (Proteintech, Wuhan, China, 1:10000) overnight at $4^{\circ} \mathrm{C}$, followed by horseradish peroxidase (HRP) conjugated secondary antibody (Cell Signaling Technology, Boston, USA, 1:5000) for $1 \mathrm{~h}$. The signal intensity was quantitatively assessed with a standard protocol for electrochemiluminescence (New Cell \& Molecular Biotech Co, Jiangsu, China) and was normalized to $\beta$-actin.

\section{Statistical analysis}

The Statistical analysis was performed in GraphPad Prism version 6.0 (GraphPad, La Jolla, CA, USA). A 2 -tailed t-test was performed between the two groups. The comparison among multiple groups was carried out by a one-way ANOVA followed by the Tukey's multiple comparison test. The correlation was calculated with the Spearman's rank correlation test. The results are presented as the mean \pm S.E.M. or mean \pm S.D. The hierarchical cluster analysis clustered the expressions of differentially expressed mRNAs and miRNAs based on the Euclidean distance and the complete-linkage clustering algorithm. The right-tailed Fisher's exact test was performed according to the IPA to calculate a $P$-value and identify statistically significant pathways and networks associated with the proteins identified in the study. The IPA z-score algorithm was used to predict the activation state for a given biological function. A z-score $\geq 2$ means a significantly increased function, whereas a $\mathrm{z}$-score $\leq 2$ indicates that a function is significantly decreased. For all the analyses, a threshold of $P<0.05$ was considered to be statistically significant.

\section{Results}

Differentially expressed microRNAs and mRNAs in placentas of GDM patients

The transcriptional mRNA and miRNA changes were identified by RNA-seq analysis of 8 placental samples from the GDM group and 8 samples from the control group (Table 1). We obtained approximately about 20 million read pairs and detected more than 23,000 expressed genes in each sample. We further compared the expression profiles of the GDM and control groups. In total, 26255 and 1402 annotated mRNAs and miRNAs were identified, respectively. Among these, 281 mRNAs $(P<0.05$; absolute value fold change $\geq 2)$ and 32 miRNAs $(P<0.05$; absolute value fold change $\geq 2)$ were significantly deregulated (Table S2). Hierarchical clustering was performed with the datasets of differentially expressed mRNAs and miRNAs. The mRNAs and miRNAs expression patterns of the GDM and control samples were clustered separately after unsupervised clustering (Fig. 1A and 1B), suggesting a profound effect of GDM on placental mRNA/miRNA expression.

Table 1. Clinical characteristics of subjects with GDM and control

\begin{tabular}{lcccccc}
\hline \multirow{2}{*}{ Characteristics } & \multicolumn{3}{c}{ Initial RNA-seq set } & \multicolumn{3}{c}{ Validation set } \\
& Control $(\mathrm{n}=8)$ & GDM $(\mathrm{n}=8)$ & P-value & Control $(\mathrm{n}=18)$ & GDM $(\mathrm{n}=20)$ & P-value \\
\hline Age (year) & $31.00 \pm 1.035$ & $32.88 \pm 1.043$ & 0.2226 & $30.8 \pm 0.7931$ & $32.75 \pm 0.70290 .7691$ \\
Gestational age (day) & $273.25 \pm 4.46$ & $272.00 \pm 4.54$ & 0.587 & $272.2 \pm 1.361$ & $272.8 \pm 0.9420$ & 0.6995 \\
BMI $\left(\mathrm{kg} / \mathrm{m}^{2}\right)$ & $20.79 \pm 0.73$ & $22.46 \pm 3.19$ & 0.170 & $20.76 \pm 0.2657$ & $22.05 \pm 0.5872$ & 0.0619 \\
OGTT 0 h & $4.00 \pm 0.39$ & $4.48 \pm 0.37$ & 0.025 & $3.917 \pm 0.07715$ & $4.856 \pm 0.19710 .0001$ \\
OGTT 1 h & $8.04 \pm 0.75$ & $10.94 \pm 1.12$ & $<0.001$ & $7.894 \pm 0.1476$ & $10.73 \pm 0.4123<0.001$ \\
OGTT 2 h & $6.69 \pm 1.34$ & $9.73 \pm 0.75$ & $<0.001$ & $6.362 \pm 0.3403$ & $9.385 \pm 0.4087<0.001$ \\
HbA1c (\%) & $5.11 \pm 0.18$ & $5.01 \pm 0.25$ & 0.372 & $4.889 \pm 0.1022$ & $5.085 \pm 0.13850 .2707$ \\
Birth weight(g) & $3575.63 \pm 112.04$ & $3386.25 \pm 311.41$ & 0.128 & $3504 \pm 64.19$ & $3514 \pm 71.71$ & 0.9219 \\
Placenta weight (g) & $608.0 \pm 20.59$ & $633.5 \pm 18.01$ & 0.3636 & $627.8 \pm 15.89$ & $636.5 \pm 14.24$ & 0.6843 \\
Weight gain (kg) & $15.55 \pm 5.64$ & $11.18 \pm 4.07$ & 0.097 & $17.28 \pm 1.309$ & $12.19 \pm 1.233$ & 0.0075 \\
Male offspring (n) & 4 & 4 & & 10 & 10 & \\
Female offspring (n) & 4 & 4 & & 8 & 10 \\
\hline
\end{tabular}


A

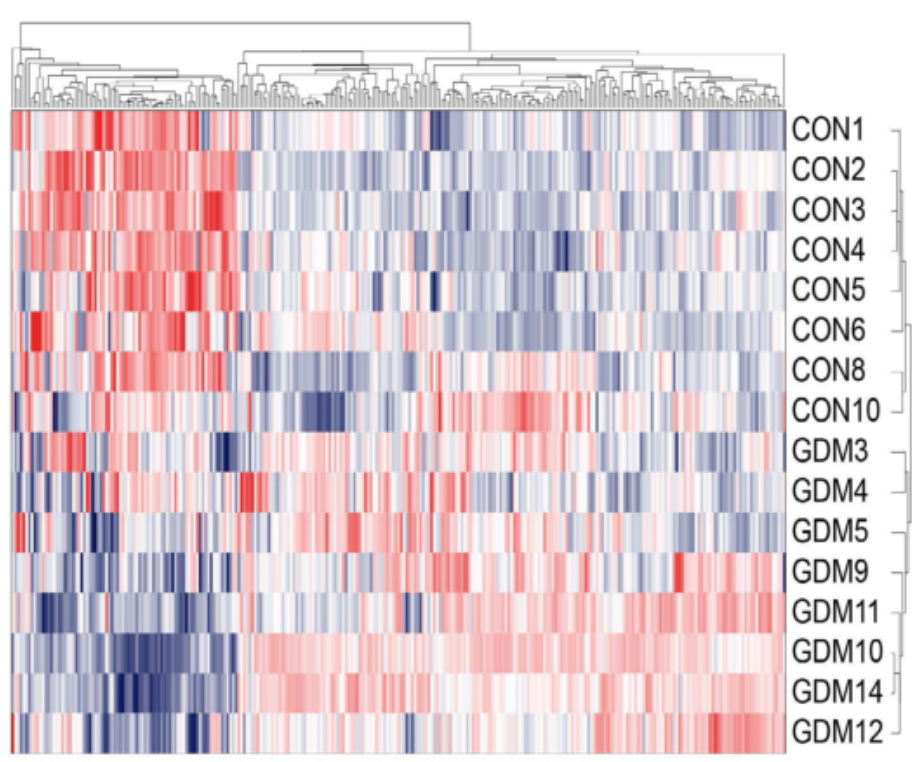

B

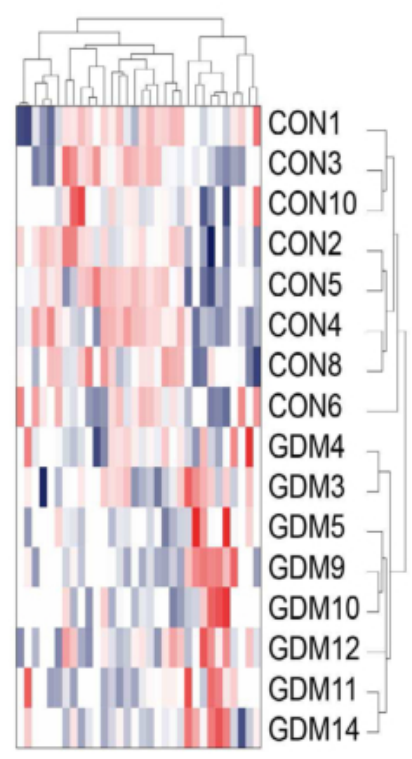

C

D
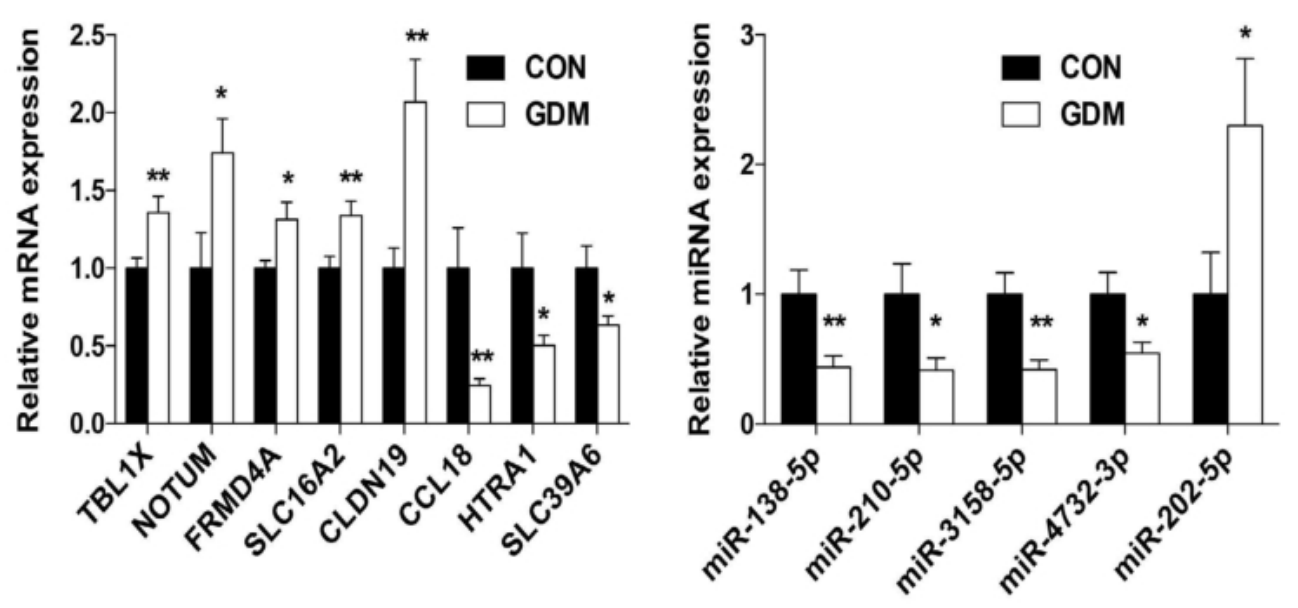

Fig. 1. Hierarchical cluster analysis and validation of differentially expressed mRNAs and miRNAs in GDM. (A) Hierarchical clustering presentation of the significantly altered mRNA and (B) miRNA profiles identified in GDM. Up-regulated and down-regulated mRNAs/miRNAs are indicated in shades of red and blue, respectively. (C-D) The differentially expressed mRNAs and miRNAs were validated by qPCR with independent GDM placentas $(n=20)$ and control placentas $(n=18)$. Data are shown as the mean \pm S.E.M. ${ }^{* *} \mathrm{P}<0.01,{ }^{*} \mathrm{P}<0.05$.

The differentially expressed mRNAs and miRNAs in GDM were validated by quantitative PCR (qPCR) based on independent 20 GDM placentas and 18 control placentas (Table 1; Fig. 1C and 1D). Ten mRNAs and 6 miRNAs, which were identified to be differentially expressed in GDM, were selected. Among them, 8 mRNAs and 5 miRNAs were validated by qPCR, including 5 upregulated mRNAs (TBL1X, NOTUM, FRMD4A, SLC16A2 and CLDN19), 1

\section{KARGER}


Fig. $\quad 2$.

Downstream effects analysis of differentially expressed mRNAs and miRNAs and m R N A - mi R N A pairs interaction network analysis in GDM. (A) We used the z-score algorithm to identify biological functions that are expected to be increased/ decreased in GDM compared to the control according to the mRNA and miRNA sequencing data. To enhance the stringency of our analysis, we considered only the functions with the fold change $\geq 2$ or $\leq 1 / 2$ (the increased result is indicated by the orange bar; the decreased result is indicated by the blue bar). The P-value (red dots), calculated with the Fischer's exact test, reflects the likelihood that the association between a set of molecules in our dataset and a biological function is sign if ic ant. (B) The most s ig n ificant b i o log i c a l network of "Cellular Development and Function, Organ Morphology, Organismal Development" was generated based on paired miRNAs-mRNAs. Upregulated mRNAs are indicated in red, while downregulated miRNAs are in green.

A

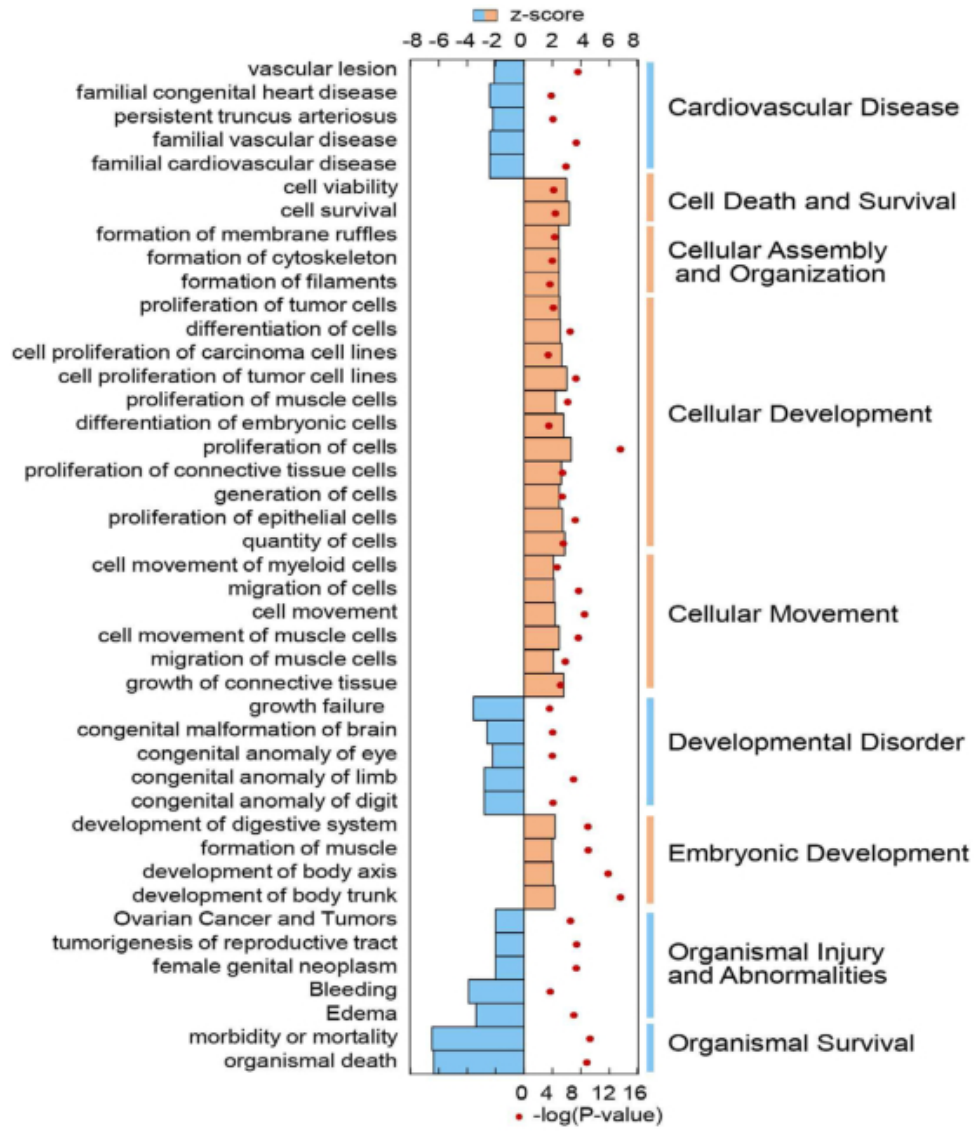

B

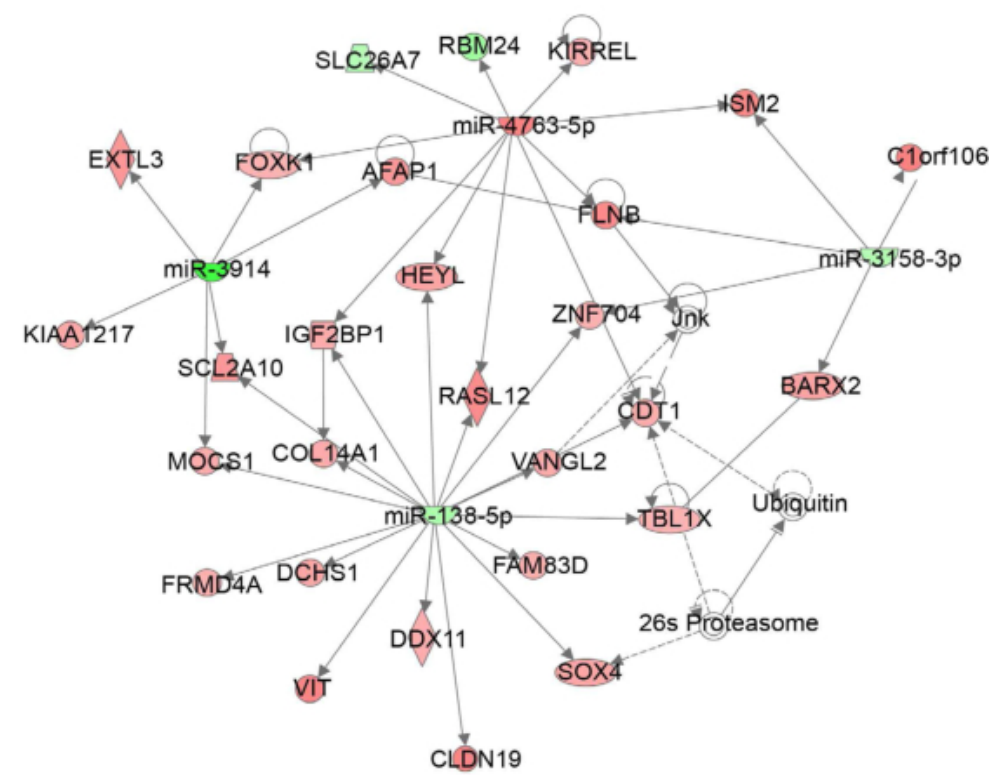




\section{Cellular Physiology Cell Physiol Biochem 2018;51:630-646 \begin{tabular}{ll|l} 
and Biochemistry Published online: 21 November 2018 & $\begin{array}{l}\text { (c) } 2018 \text { The Author(s). Published by S. Karger AG, Basel } \\
\text { www.karger.com/cpb }\end{array}$ \\
\hline
\end{tabular} \\ Ding et al.: Transcriptome Profile of GDM Placenta}

upregulated miRNA (miR-202-5p), 3 down-regulated mRNAs (CCL18, HTRA1, SLC39A6) and 4 down-regulated miRNAs (miR-138-5p, miR-210-5p, miR-3158-5p and miR-4732-3p). Only 2 mRNAs (BEX1, EPYC) and 1 miRNA (miR-5096) were not confirmed.

Integrative analysis of the differentially expressed mRNAs and miRNAs identified in GDM placentas

We performed functional analysis with the Ingenuity Pathway Analysis (IPA, Ingenuity ${ }^{\circledR}$ Systems) to elucidate the influences of differentially expressed mRNAs and miRNAs on biological functions. To our knowledge, this is the first time that molecules from miRNA-seq and mRNA-seq were simultaneously combined with annotated functions in GDM studies. The IPA identified the top biological processes (Table S3) and canonical pathways (Table S4) enriched among the differentially expressed mRNAs and miRNAs. The downstream effects analysis with two metrics, namely, the z-score and $P$-value, repeatedly predicted the significant upregulation of "cellular development" and "cellular movement". The increased "proliferation of cells" (z-score $=3.32 ; P=2.56 \times 10^{-14}$ ) and decreased "morbidity or mortality" (z-score=-6.494; $P=4.8 \times 10^{-10}$ ) were the most affected subcategories (Fig. $2 \mathrm{~A}$ ).

To address the functional significance of the differentially expressed miRNAs and putative target mRNAs, paired miRNAs-mRNAs were identified with the IPA based on the miRNA target databases (TargetScan, TarBase, miRecords, and Ingenuity Expert Findings) and expression direction. We detected 91 negatively expressed mRNA targets for 13 miRNAs (Table S5 based on IPA) and then further generated molecular interaction networks based on molecule connectivity and ranked them according to their scores to dissect the biological relationships of the above miRNA-mRNA pairs (Table S6). As expected, the most enriched network was related to "Cellular Development and Function, Organ Morphology, Organismal Development" (score=77) (Fig. 2A). This result was consistent with the downstream effects analysis in which the alterations in placental development and morphology were the major phenotypic changes detected in GDM. Notably, miR-138-5p was a central "node" in the network (Fig. 2B), with the maximum number of nodes, and was negatively co-expressed with its predicted targets (Fig. S1).

Overexpression of miR-138-5p inhibited the migration and proliferation of HTR-8/SVneo

We then investigated whether miR-138-5p was involved in the migratory and proliferative capacity of trophoblasts. For this purpose, we used a first-trimester human EVT-derived cell line named HTR-8/SVneo (HTR-8). The cells were transfected with miR138-5p mimic or miR-138-5p inhibitor. The wound healing (Fig. 3A) and transwell migration (Fig. 3B) assays demonstrated that overexpression of miR-138-5p decreased the migratory ability of HTR-8 cells, whereas the inhibition of miR-138-5p increased the migratory ability of HTR-8 cells compared to the corresponding negative control. To further confirm the role of miR-138-5p in trophoblast proliferation, CCK8 assays were conducted. The results revealed that miR-138-5p overexpression significantly reduced the proliferation of HTR8 cells, whereas the inhibition of miR-138-5p clearly increased proliferation compared to the corresponding negative control (Fig. 3C). These results suggested that miR-138-5p is required in the migration and proliferation of human trophoblasts.

TBL1X was validated as a target of miR-138-5p

$T B L 1 X$ is a putative target gene of miR-138-5p. To further verify TBL1X targeting by miR-138-5p, the dual luciferase assay was carried out in HTR-8 and HEK293T cells. The relative luciferase activity of the wild-type reporter (WT) cotransfected with miR-138-5p mimic was reduced to $68 \%$ in HTR-8 and $67 \%$ in HEK293T cells $(P<0.01$, Fig. 4 A). There was no significant change in the luciferase activity in the construct with the mutant TBL1X 3 'UTR (Mut). The above results suggested that miR-138-5p could target TBL1X.

We assessed the correlation between miR-138-5P and the TBL1X protein level in HTR-8 cells after transfection with miR-138-5p mimic, miR-138-5p inhibitor or negative control. As predicted, miR-138-5p significantly suppressed TBL1X protein expression compared to 


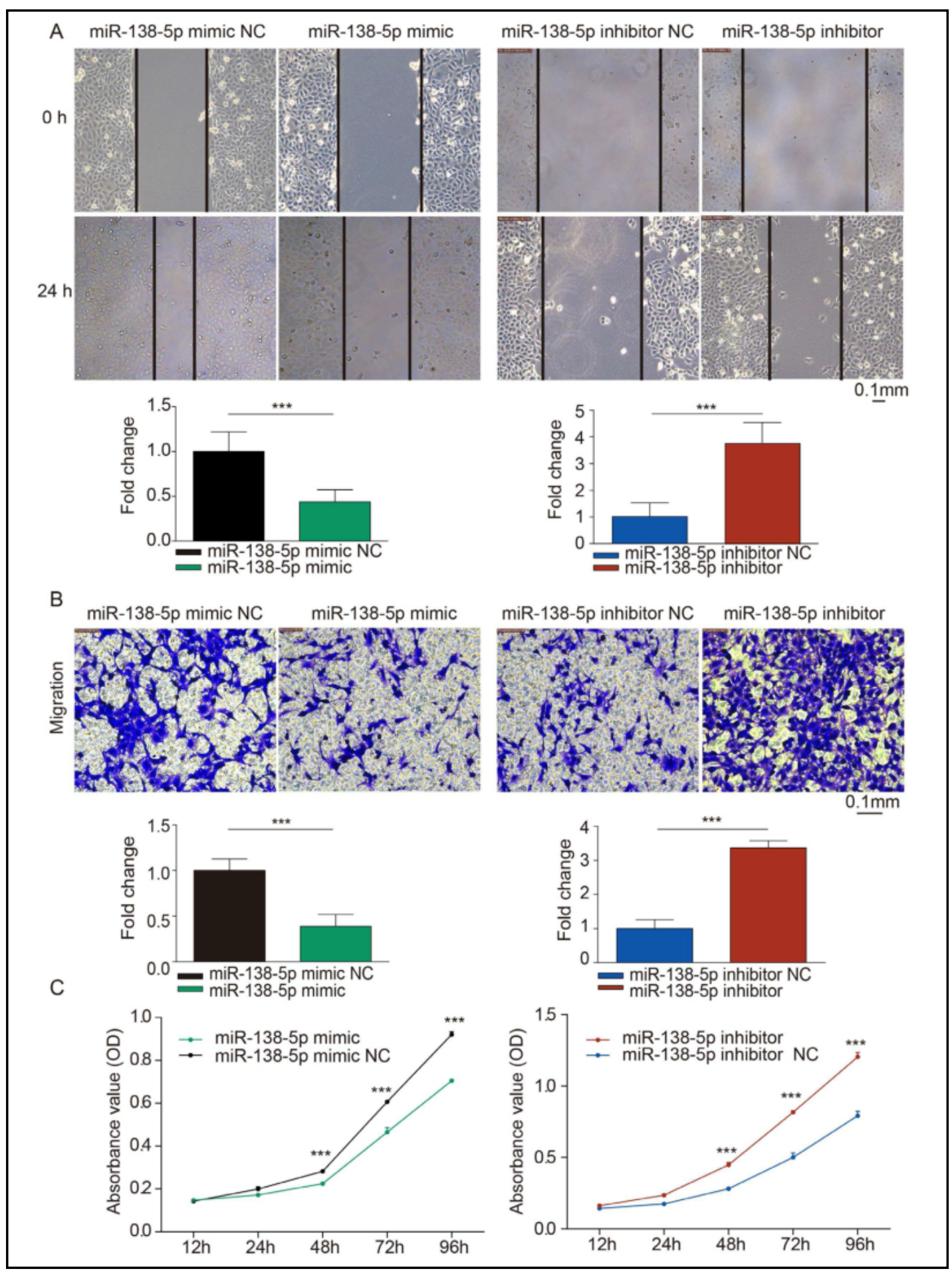

Fig. 3. miR-138-5p regulates migration and proliferation in trophoblasts. (A) HTR8 cells were transfected with miR-138-5p mimic, miR-138-5p inhibitor and their corresponding negative controls (NC). The migration ability was measured after $24 \mathrm{~h}$ in the wound-healing assay. (B) Overexpression of miR-138-5p in HTR8 cells significantly decreased the number of migratory cells compared with that of the NC. Decreased of miR-138-5p drastically increased the number of migratory cells compared with that of the NC. (C) Overexpression of miR-138-5p in HTR8 cells resulted in reduced proliferation ability compared with that of the NC (left). Knockdown of the miR-138-5p in HTR8 cells significantly increased the proliferation ability compared with that of the NC. Data are shown as the mean \pm SEM. ${ }^{* *} \mathrm{P}<0.01,{ }^{* * *} \mathrm{P}<0.001$.

\section{KARGER}




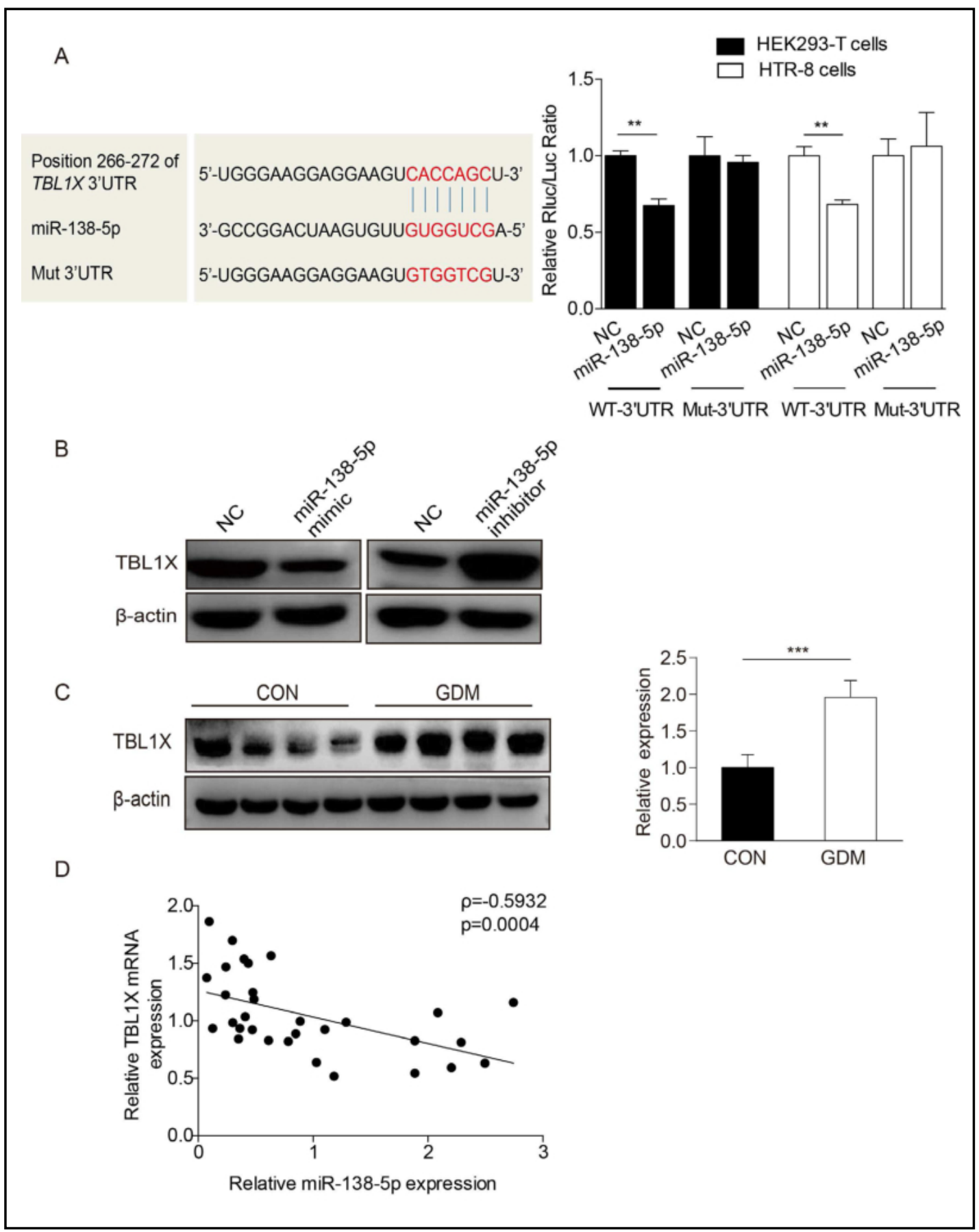

Fig. 4. miR-138-5p represses TBL1X expression in trophoblasts. (A) The miR-138-5p-binding site in the 3'-UTR of TBL1X is illustrated in the diagram. The luciferase reporter vector (TBL1X wild-type or TBL1X mutant) was co-transfected with the miR-138-5p mimic or the corresponding negative control into HTR8 cells. The luciferase activity was measured after $24 \mathrm{~h}$. The transfection efficiency differences were normalized to the firefly luciferase activity. (B) Western blotting analyses of TBL1X expression in HTR8 cells transfected with miR-138-5p mimic, miR-138-5p inhibitor and corresponding negative control. (C) The protein level of TBL1X was significantly downregulated in the GDM placenta. (D) The TBL1X mRNA expression was negatively correlated with the expression of miR-138-5p. ${ }^{* *} \mathrm{P}<0.01,{ }^{* * *} \mathrm{P}<0.001$. 


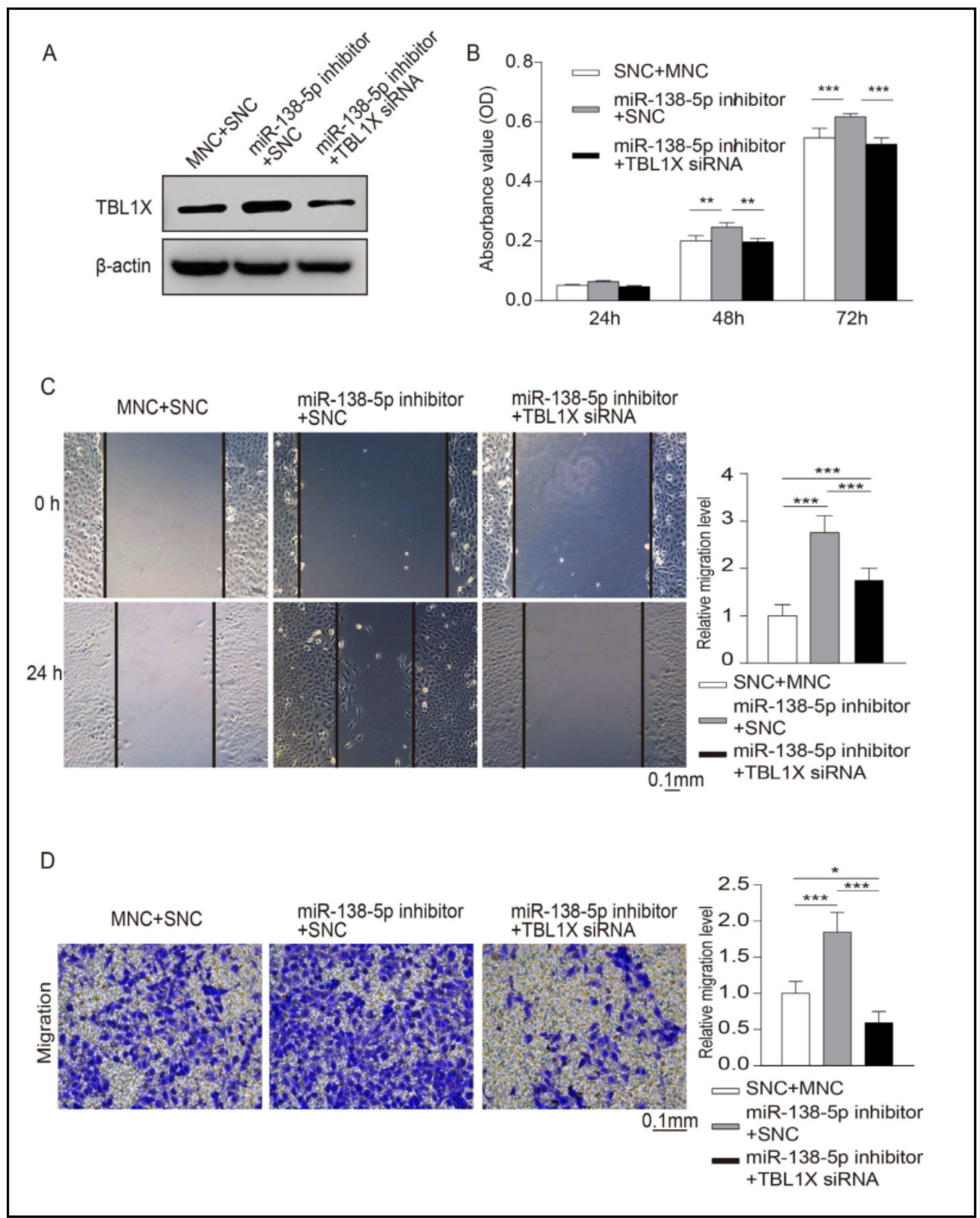

Fig. 5. The migratory and proliferative effects of miR-138-5p inhibition are reversed by the knockdown of TBL1X in trophoblasts. (A) Western blot analysis of TBL1X in HTR-8 cells after co-transfection with miR138-5p inhibitor and TBL1X siRNA. (B) Cell proliferation was inhibited after co-transfection with miR138-5p inhibitor and TBL1X siRNA. (C) The co-transfection of TBL1X siRNA reduced the scratch width 48 $\mathrm{h}$ after transfection. (D) Cell migration was reduced after co-transfection with miR-138-5p inhibitor and TBL1X siRNA. Data are shown as the mean \pm SEM. ${ }^{* *} \mathrm{P}<0.01,{ }^{* * *} \mathrm{P}<0.001$. MNC, miR-138-5p inhibitor negative control; SNC, TBL1X siRNA negative control. 
the corresponding negative control, whereas the protein levels of TBL1X were up-regulated upon the inhibition of miR-138-5p compared to the corresponding negative control (Fig. 4B). After verifying the correlation between miR-138-5p and TBL1X expression in HTR8 cells, qPCR and Western blotting were used to evaluate their relationship in placental tissues. The results showed that the mRNA $(P<0.01$, Fig. $1 \mathrm{C})$ and protein $(P<0.001$, Fig. $4 \mathrm{C})$ levels of TBL1X were significantly up-regulated in GDM placentas. A significant negative correlation between the expression of miR-138-5p and TBL1X mRNA was also detected $(\rho=-$ $0.59, P<0.001$, Fig. 4D).

\section{The inhibitory function of miR-138-5p was exerted via the regulation of TBL1X}

To investigate whether the down-regulated TBL1X could rescue the promoting effect of miR-138-5p inhibition, we co-transfected miR-138-5p inhibitor together with TBL1X siRNA into HTR-8 cells. The results showed that TBL1X siRNA reduced the ability of miR-138-5p inhibitor to upregulate TBL1X protein expression (Fig. 5A). After co-transfecting TBL1X siRNA and miR-138-5p inhibitor, the migratory ability of HTR-8 cells was significantly inhibited in the wound healing and transwell migration assays (Fig. 5C and 5D). Additionally, the CCK8 assay revealed that the co-transfection of TBL1X siRNA reduced the increased proliferation promoted by the transfection of miR-138-5p inhibitor after $48 \mathrm{~h}$ (Fig. 5B). Taken together, these findings indicated that miR-138-5p could inhibit the migration and proliferation of HTR-8 trophoblasts by targeting TBL1X.

miR-138-5p and TBL1X were correlated with placental weights in pregnant women with GDM

Placental weight and the incidence of macrosomia have been reported to increase in GDM [32]. To confirm the macroscopic and histological alterations in the GDM placenta, we analyzed the data of 14, 701 pregnant women recruited from our hospital (from January to December 2016) (Table 2). The results indicated that the weight of the GDM placenta was significantly heavier than that of the normal placenta ( $625 \mathrm{~g}$ vs. $620 \mathrm{~g}, P<0.001$ ). The incidence of large for gestational age (LGA) was also higher in pregnant women with GDM than in those without GDM $(11.57 \%$ vs. $8.19 \%, P<0.001)$. We then examined whether miR138-5p or TBL1X expression was associated with placental weight in GDM pregnancy. Our result showed that the expression of miR-138-5p was negatively correlated with placental weight (Fig. 6A). Decreased miR-138-5p expression in the placenta was significantly correlated to increased placental weight $(\rho=-0.51, P<0.01)$. However, the expression of TBL1X was positively correlated with placental weight (Fig. 6B, $\rho=0.38, P=0.05$ ).

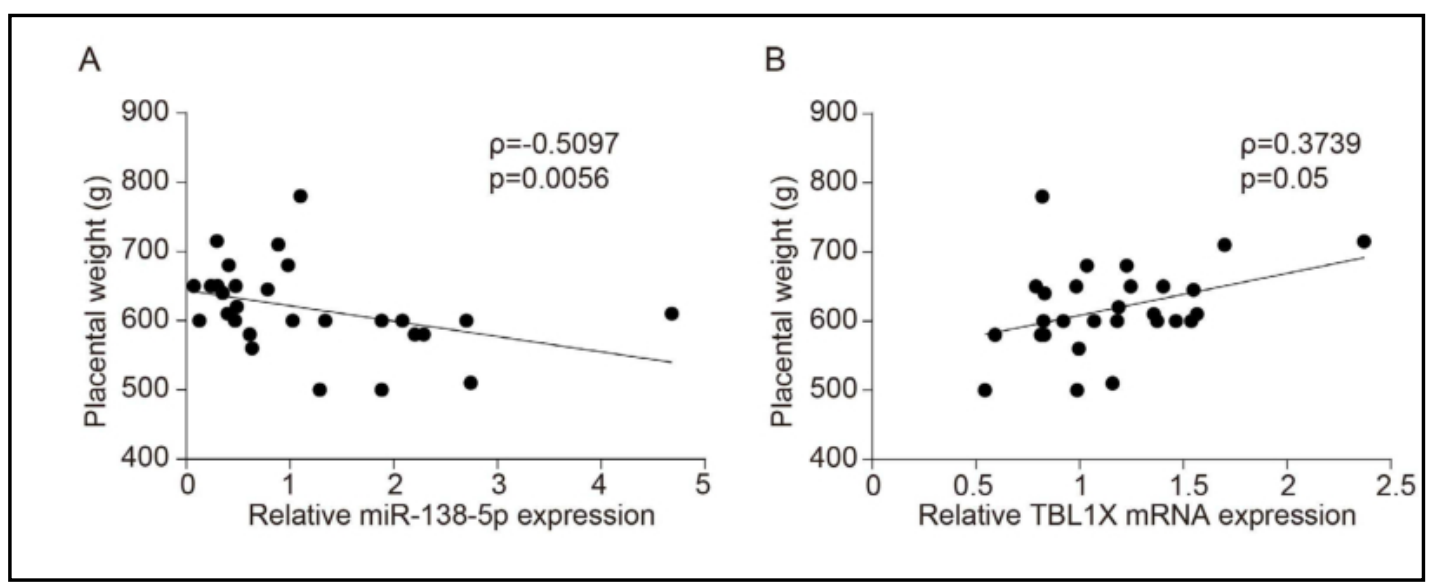

Fig. 6. Correlation between the expression of placental miR-138-5p and TBL1X and placental weight. (A)The expression of placental miR-138-5p is negatively correlated with the placental weight. (B) The expression of placental TBL1X is positively correlated with the placental weight. 


\section{Cellular Physiology Cell Physiol Biochem 2018;51:630-646 \begin{tabular}{l|l|l} 
and Biochemistry Published online: 21 November 2018 & $\begin{array}{l}\text { (c) } 2018 \text { The Author(s). Published by S. Karger AG, Basel } \\
\text { www.karger.com/cpb }\end{array}$ \\
\hline
\end{tabular} \\ Ding et al.: Transcriptome Profile of GDM Placenta}

\section{Discussion}

Due to their ability to posttranscriptionally regulate nearly $60 \%$ of the genes in human genome, miRNAs are known to influence many biological processes in the pathogenesis and development of human disease [33],[34]. It is necessary to identify target mRNAs regulated by miRNAs in order to elucidate the exact role of the specific miRNAs. In this study, we performed RNA-seq to investigate the differentially expressed mRNAs and miRNAs in 8 GDM placentas compared with 8 normal placentas and identified the aberrantly expressed miRNA-mRNA pairs in GDM. To our knowledge, this study is the first integrative analysis of miRNA and mRNA expression profiles in GDM placentas.

GDM is a complication of pregnancy with aberrant placental functions. The appropriate proliferation, migration and differentiation of trophoblasts are directly related to hormone secretion, vascularization, and the immunological interface $[35,36]$. At the morphological level, previous studies have shown that the placentas are heavier and that the placental/birth weight ratio is higher in GDM (PW/BW ratio) $[37,38]$. Our study based on 14701 pregnant women also indicated that the placental weight and PW/BW ratio were significantly increased in GDM. The incidence of LGA was also elevated in GDM (Table 2). Clinical and experimental data from mouse models indicated that the PW/BW ratio and placental weight were often used as an indicative maker of altered placental nutrient transfer [38]. Furthermore, a high number of villous cytotrophoblasts were found in the GDM placenta at the cellular level [39]. Unek et al. reported that the expression levels of cell proliferation markers, Ki67 and PCNA, were significantly increased in trophoblasts from hyperglycemia placentas compared to the controls [40]. Thus, a large number of proliferative trophoblasts may be associated with morphological changes in the GDM placenta.

What is the underlying cause of the increased placental weight and trophoblast proliferation? A previous study has indicated the remarkable similarities between the proliferative, migratory and invasive properties of trophoblasts and cancer cells [41]. miRNAs have been shown to participate in the carcinogenesis of several types of tumors, and they can impair cellular movement, invasion, growth and proliferation, thus promoting the development of tumors [42]. miRNAs are also expressed in the placenta and have been reported in pregnancy complications including GDM and preeclampsia [21, 43]. Fu et al. identified a series of miRNAs that were involved in dysregulated placental development and function [44]. In our study, the downstream effects analysis among the differentially expressed mRNAs and miRNAs repeatedly predicted the increased proliferation of cells. Furthermore, the biological relationship between the filtered miRNA-mRNA pairs also revealed significant enrichment in "Cellular Development and Function, Organ Morphology, and Organismal Development". Therefore, these results not only identified that cell proliferation was an important phenotypic abnormality differentiated between the GDM and control groups, but also indicated that miRNAs functioned as key regulators in the pathology of GDM. Obviously, miR-138-5p was a core "node" in the miRNA-mRNA network and was negatively co-expressed with its predicted targets. Interestingly, the downregulation of miR138-5p plays an important role in various cancers. For example, it was reported that miR138-5p could inhibit BIRC5 translation and suppress the BIRC5-meditated carcinogenesis of bladder cancer [45]. Similarly, miR-138-5p was also downregulated in human colorectal cancer (CRC) and dramatically promoted CRC cell growth and tumorigenesis by targeting PD-L1 [46]. In this study, we also found that miR-138-5p was downregulated in the GDM 


\section{Cellular Physiology Cell Physiol Biochem 2018;51:630-646 \begin{tabular}{ll|l} 
and Biochemistry & $\begin{array}{l}\text { DOl: 10.1159/000495319 } \\
\text { Published online: 2T November } 2018\end{array}$ & $\begin{array}{l}\text { O 2018 The Author(s). Published by S. Karger AG, Basel } \\
\text { ww.karger.com/cpb }\end{array}$ \\
\cline { 2 - 3 } &
\end{tabular} \\ Ding et al.: Transcriptome Profile of GDM Placenta}

placenta. To investigate the role of miR-138-5p in the pathology of the GDM placenta, we suppressed miR-138-5p in HTR-8 cells and found that the proliferation and migration of trophoblasts were increased.

TBL1X is a potential target of miR-138-5p, as predicted by the IPA's microRNA Target Filter tool. TBL1X functions as an oncogene in the proliferation and tumorigenesis of hepatocellular carcinoma (HCC) cells through activating WNT/ $\beta$-catenin signaling [47]. The bioinformatics approaches in our study revealed that the miRNA signature identified gene targets involved in the WNT/ $\beta$-catenin signaling pathway. According to the present literature, $\mathrm{WNT} / \beta$-catenin signaling is suggestive of its crucial role for physiological process of human placenta. It is involved in the regulation of cell fusion, proliferation, invasion and differentiation [48]. Moreover, WNT/ $\beta$-catenin signaling has been implicated in the regulation of insulin resistance [49]. In our study, TBL1X mRNA and protein expression were significantly increased in the GDM placenta. Therefore, we inferred that aberrant expression of miR-138-5p contributed to the abnormal growth of the placenta by enhancing the proliferation of trophoblasts by targeting TBL1X.

\section{Conclusion}

In summary, this study presents the first integrated analysis of miRNA-mRNA expression in GDM placenta. Moreover, this study is the first to provide evidence for the role of the miR$138-5 p$ in the proliferation and migration of trophoblasts via TBL1X targeting. The findings in this study indicate that the integrated miRNA-mRNA strategy can be used to identify novel mechanisms for GDM.

\section{Abbreviations}

GDM (Gestational diabetes mellitus); microRNA (miRNA); RNA-seq (RNA sequencing); qPCR (quantitative PCR); hPL (human placental lactogen); IVF (in vitro fertilization); IPA (Ingenuity Pathway Analysis); HCC (hepatocellular carcinoma); HTR-8 (HTR-8/SVneo cells); FBS (fetal bovine serum); PW/BW (ratio, placental/birth weight ratio).

\section{Acknowledgements}

We thank all the participants in this research. This work is supported by the National Natural Science Foundation of China [NO. 81471516; 81401219; 81501276; 81501274], the National Key Research and Development Program of China [NO. 2016YFC1000203], the Shanghai Municipal Commission of Science and Technology Program [NO. 15411966700], the Shanghai Municipal Commission of Health and Family Planning [NO. 15GWZK0701; 20154Y0039], the Municipal Human Resources Development Program for Outstanding Young Talents in Medical and Health Sciences in Shanghai [NO.2018YQ39], and the Combined Engineering and Medical Project of Shanghai Jiao Tong University [NO. YG2017MS39].

\section{Disclosure Statement}

The authors declare that there are no competing financial interests. 


\section{Cellular Physiology Cell Physiol Biochem 2018;51:630-646

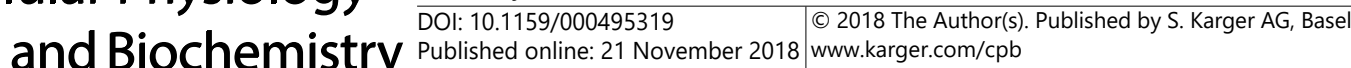

Ding et al.: Transcriptome Profile of GDM Placenta

\section{References}

1 American Diabetes A: Standards of medical care in diabetes--2014. Diabetes care 2014;37 Suppl 1:S14-80.

2 Tutino GE, Tam WH, Yang X, Chan JC, Lao TT, Ma RC: Diabetes and pregnancy: perspectives from Asia. Diabetic medicine : a journal of the British Diabetic Association 2014;31:302-318.

-3 Group HSCR: Hyperglycemia and Adverse Pregnancy Outcome (HAPO) Study: associations with neonatal anthropometrics. Diabetes 2009;58:453-459.

-4 Amrithraj AI, Kodali A, Nguyen L, Teo AKK, Chang CW, Karnani N, Ng KL, Gluckman PD, Chong YS, Stunkel W: Gestational Diabetes Alters Functions in Offspring's Umbilical Cord Cells With Implications for Cardiovascular Health. Endocrinology 2017;158:2102-2112.

-5 Holder T, Giannini C, Santoro N, Pierpont B, Shaw M, Duran E, Caprio S, Weiss R: A low disposition index in adolescent offspring of mothers with gestational diabetes: a risk marker for the development of impaired glucose tolerance in youth. Diabetologia 2014;57:2413-2420.

-6 Ding GL, Wang FF, Shu J, Tian S, Jiang Y, Zhang D, Wang N, Luo Q, Zhang Y, Jin F, Leung PC, Sheng JZ, Huang HF: Transgenerational glucose intolerance with Igf2/H19 epigenetic alterations in mouse islet induced by intrauterine hyperglycemia. Diabetes 2012;61:1133-1142.

7 Kuhl C: Etiology and pathogenesis of gestational diabetes. Diabetes care 1998;21 Suppl 2:B19-26.

8 Muralimanoharan S, Maloyan A, Myatt L: Mitochondrial function and glucose metabolism in the placenta with gestational diabetes mellitus: role of miR-143. Clinical science 2016;130:931-941.

-9 Fetita LS, Sobngwi E, Serradas P, Calvo F, Gautier JF: Consequences of fetal exposure to maternal diabetes in offspring. J Clin Endocrinol Metab 2006;91:3718-3724.

10 Freemark M: Placental hormones and the control of fetal growth. The Journal of clinical endocrinology and metabolism 2010;95:2054-2057.

-11 Szukiewicz D: Cytokines in placental physiology and disease. Mediators of inflammation 2012;2012:640823.

-12 Wallace JM, Horgan GW, Bhattacharya S: Placental weight and efficiency in relation to maternal body mass index and the risk of pregnancy complications in women delivering singleton babies. Placenta 2012;33:611-618.

13 Lopez-Espinoza I, Smith RF, Gillmer M, Schidlmeir A, Hockaday TD: High levels of growth hormone and human placental lactogen in pregnancy complicated by diabetes. Diabetes research 1986;3:119-125.

-14 Lassance L, Haghiac M, Minium J, Catalano P, Hauguel-de Mouzon S: Obesity-induced down-regulation of the mitochondrial translocator protein (TSPO) impairs placental steroid production. The Journal of clinical endocrinology and metabolism 2015;100:E11-18.

15 Vejrazkova D, Vcelak J, Vankova M, Lukasova P, Bradnova O, Halkova T, Kancheva R, Bendlova B: Steroids and insulin resistance in pregnancy. The Journal of steroid biochemistry and molecular biology 2014;139:122-129.

16 Aires MB, Dos Santos AC: Effects of maternal diabetes on trophoblast cells. World journal of diabetes 2015;6:338-344.

17 Nelissen EC, van Montfoort AP, Dumoulin JC, Evers JL: Epigenetics and the placenta. Human reproduction update 2011;17:397-417.

18 Ambros V: The functions of animal microRNAs. Nature 2004;431:350-355.

-19 Mouillet JF, Ouyang Y, Coyne CB, Sadovsky Y: MicroRNAs in placental health and disease. American journal of obstetrics and gynecology 2015;213:S163-172.

$>20$ Li J, Song L, Zhou L, Wu J, Sheng C, Chen H, Liu Y, Gao S, Huang W: A MicroRNA Signature in Gestational Diabetes Mellitus Associated with Risk of Macrosomia. Cellular physiology and biochemistry : international journal of experimental cellular physiology, biochemistry, and pharmacology 2015;37:243-252.

21 Shi Z, Zhao C, Guo X, Ding H, Cui Y, Shen R, Liu J: Differential expression of microRNAs in omental adipose tissue from gestational diabetes mellitus subjects reveals miR-222 as a regulator of ERalpha expression in estrogen-induced insulin resistance. Endocrinology 2014;155:1982-1990.

22 Bari MF, Ngo S, Bastie CC, Sheppard AM, Vatish M: Gestational diabetic transcriptomic profiling of microdissected human trophoblast. The Journal of endocrinology 2016;229:47-59.

23 Cheng C, Guo L, Lu L, Xu X, Zhang S, Gao J, Waqas M, Zhu C, Chen Y, Zhang X, Xuan C, Gao X, Tang M, Chen F, Shi H, Li H, Chai R: Characterization of the Transcriptomes of Lgr5+ Hair Cell Progenitors and Lgr5Supporting Cells in the Mouse Cochlea. Front Mol Neurosci 2017;10:122. 


\section{Cellular Physiology Cell Physiol Biochem 2018;51:630-646 and Biochemistry DOl: 10.1159/000495319 2018 (0) 2018 The Author(s). Published by S. Karger AG, Basel

Ding et al.: Transcriptome Profile of GDM Placenta

-24 Lohse M, Bolger AM, Nagel A, Fernie AR, Lunn JE, Stitt M, Usadel B: RobiNA: a user-friendly, integrated software solution for RNA-Seq-based transcriptomics. Nucleic acids research 2012;40:W622-627.

-25 Trapnell C, Williams BA, Pertea G, Mortazavi A, Kwan G, van Baren MJ, Salzberg SL, Wold BJ, Pachter L: Transcript assembly and quantification by RNA-Seq reveals unannotated transcripts and isoform switching during cell differentiation. Nature biotechnology 2010;28:511-515.

-26 Martin M: Cutadapt removes adapter sequences from high-throughput sequencing reads. 2011 2011;17

-27 Li H, Durbin R: Fast and accurate short read alignment with Burrows-Wheeler transform. Bioinformatics 2009;25:1754-1760.

-28 Kozomara A, Griffiths-Jones S: miRBase: annotating high confidence microRNAs using deep sequencing data. Nucleic acids research 2014;42:D68-73.

29 Anders S, Huber W: Differential expression analysis for sequence count data. Genome biology 2010;11:R106.

-30 Xu GF, Zhang JY, Pan HT, Tian S, Liu ME, Yu TT, Li JY, Ying WW, Yao WM, Lin XH, Lv Y, Su WW, Ye XQ, Zhang FH, Pan JX, Liu Y, Zhou CL, Zhang D, Liu XM, Zhu YM, Sheng JZ, Huang HF: Cardiovascular dysfunction in offspring of ovarian-hyperstimulated women and effects of estradiol and progesterone: a retrospective cohort study and proteomics analysis. J Clin Endocrinol Metab 2014;99:E2494-2503.

31 Kramer A, Green J, Pollard J, Jr., Tugendreich S: Causal analysis approaches in Ingenuity Pathway Analysis. Bioinformatics 2014;30:523-530.

32 Ramos A, Caimari F, Pujol IM, Garcia-Patterson A, Ginovart G, Adelantado JM, Corcoy R: In women with gestational diabetes mellitus factors influencing growth have a larger effect on placental weight than on birth weight. European journal of obstetrics, gynecology, and reproductive biology 2016;202:60-65.

-33 Friedman RC, Farh KK, Burge CB, Bartel DP: Most mammalian mRNAs are conserved targets of microRNAs. Genome research 2009;19:92-105.

-34 Hammond SM: An overview of microRNAs. Advanced drug delivery reviews 2015;87:3-14.

-35 Mannik J, Vaas P, Rull K, Teesalu P, Laan M: Differential placental expression profile of human Growth Hormone/Chorionic Somatomammotropin genes in pregnancies with pre-eclampsia and gestational diabetes mellitus. Molecular and cellular endocrinology 2012;355:180-187.

36 Gauster M, Desoye G, Totsch M, Hiden U: The placenta and gestational diabetes mellitus. Current diabetes reports 2012;12:16-23.

37 Huynh J, Dawson D, Roberts D, Bentley-Lewis R: A systematic review of placental pathology in maternal diabetes mellitus. Placenta 2015;36:101-114.

38 Hayward CE, Lean S, Sibley CP, Jones RL, Wareing M, Greenwood SL, Dilworth MR: Placental Adaptation: What Can We Learn from Birthweight:Placental Weight Ratio? Frontiers in physiology 2016;7:28.

-39 Fock V, Mairhofer M, Otti GR, Hiden U, Spittler A, Zeisler H, Fiala C, Knofler M, Pollheimer J: Macrophagederived IL-33 is a critical factor for placental growth. Journal of immunology 2013;191:3734-3743.

$>40$ Unek G, Ozmen A, Mendilcioglu I, Simsek M, Korgun ET: Immunohistochemical distribution of cell cycle proteins p27, p57, cyclin D3, PCNA and Ki67 in normal and diabetic human placentas. Journal of molecular histology 2014;45:21-34.

-41 Ferretti C, Bruni L, Dangles-Marie V, Pecking AP, Bellet D: Molecular circuits shared by placental and cancer cells, and their implications in the proliferative, invasive and migratory capacities of trophoblasts. Hum Reprod Update 2007;13:121-141.

42 Di Leva G, Garofalo M, Croce CM: MicroRNAs in cancer. Annual review of pathology 2014;9:287-314.

43 Luo R, Shao X, Xu P, Liu Y, Wang Y, Zhao Y, Liu M, Ji L, Li YX, Chang C, Qiao J, Peng C, Wang YL: MicroRNA-210 contributes to preeclampsia by downregulating potassium channel modulatory factor 1 . Hypertension 2014;64:839-845.

44 Fu G, Brkic J, Hayder H, Peng C: MicroRNAs in Human Placental Development and Pregnancy Complications. International journal of molecular sciences 2013;14:5519-5544.

45 Yang R, Liu M, Liang H, Guo S, Guo X, Yuan M, Lian H, Yan X, Zhang S, Chen X, Fang F, Guo H, Zhang C: miR-138-5p contributes to cell proliferation and invasion by targeting Survivin in bladder cancer cells. Molecular cancer 2016;15:82.

46 Zhao L, Yu H, Yi S, Peng X, Su P, Xiao Z, Liu R, Tang A, Li X, Liu F, Shen S: The tumor suppressor miR-138-5p targets PD-L1 in colorectal cancer. Oncotarget 2016;7:45370-45384. 


\section{Cellular Physiology Cell Physiol Biochem 2018;51:630-646}

and Biochemistry DOl: 10.1159/000495319 2018 The Author(s). Published by S. Karger AG, Basel

Ding et al.: Transcriptome Profile of GDM Placenta

47 Zeng XC, Liu FQ, Yan R, Yi HM, Zhang T, Wang GY, Li Y, Jiang N: Downregulation of miR-610 promotes proliferation and tumorigenicity and activates Wnt/beta-catenin signaling in human hepatocellular carcinoma. Molecular cancer 2014;13:261.

48 Knofler M, Pollheimer J: Human placental trophoblast invasion and differentiation: a particular focus on Wht signaling. Frontiers in genetics 2013;4:190.

-49 Daniele G, Winnier D, Mari A, Bruder J, Fourcaudot M, Pengou Z, Tripathy D, Jenkinson C, Folli F: Sclerostin and Insulin Resistance in Prediabetes: Evidence of a Cross Talk Between Bone and Glucose Metabolism. Diabetes care 2015;38:1509-1517. 